\title{
Homogeneous Observers for Projected Quadratic Partial Differential Equations
}

\author{
Sergiy Zhuk and Andrey Polyakov
}

\begin{abstract}
The paper proposes a new homogeneous observer for finite-dimensional projections of quadratic homogeneous hyperbolic PDEs with compact state space. The design relies upon new sufficient conditions for fixed-time convergence of observer's gain, described as a solution of a non-linear homogeneous matrix differential equations, towards an ellipsoid in the space of symmetric non-negative matrices. Convergence of the observer is analyzed, and a numerical convergence test is proposed: numerical experiments confirm the test on ODEs obtained by finite-difference discretization of BurgersHopf equation.
\end{abstract}

\section{INTRODUCTION}

Nonlinear filtering and observer design are fundamental in diverse fields including synchronization in complex networks, data assimilation and control engineering to name just a few. Theoretically, solution of the stochastic filtering problem for Markov diffusions is given by the so-called Kushner-Stratonovich (KS) equation [1], a stochastic Partial Differential Equation (PDE) which describes evolution of the conditional density of the states of the underlying diffusion process. For linear systems, KS equation is equivalent to the Kalman-Bucy Filter.

In contrast, deterministic state estimators (observers), including the algorithm presented in this paper, assume that errors have bounded energy and belong to a given bounding set. The optimal state estimate (or minimum energy estimate) is then defined as the minimax center of the reachability set, and temporal dynamics of the minimax center is described by a minimax filter [2]. There is a fundamental connection between stochastic filters and observers: namely, an observer can be obtained as asymptotic limit of stochastic filters associated with the "noisy" version of the state equation and observations; when "noise" disappears (e.g. noise variance approaches zero) the mean-squared error between stochastic and deterministic state estimates goes to zero, and, in fact, the conditional measure associated with the stochastic filter converges to a degenerate measure concentrated at the observer [2]. Having this in mind we consider the case of exact measurements and zero model disturbances.

Quadratic ODEs studied below can be obtained as finitedimensional projections of quadratic homogeneous hyperbolic PDEs including Euler equation in two spatial dimen-

The research is supported by the project "RECoT" of Inria NorthEuropean Associate Team Program.

Sergiy Zhuk is with IBM Research, Dublin, Ireland (sergiy.zhuk@ie.ibm.com).

Andrey Polyakov is with Inria Lille-Nord Europe, France (andrey.polyakov@inria.fr) sions [3], [4], or a finite-difference discretization of the Burgers equation in 1D [5], [6]. Such ODEs have a number of conserved quantities, e.g. energy and volume, and so all the solutions evolve on a given bounded manifold (e.g. a sphere of a certain radius) and never approach zero. In other words, the original system has no diffusion or damping, all the Lyapunov exponents equal zero and so the system is not asymptotically stable. State estimation for such systems is a challenging problem especially in the case of high dimension of the state vector. Indeed, in the latter case it is hard to implement the differential algebraic approach conventional for nonlinear systems [7], [8], [9]. Methods based on linearization, e.g. popular Extended Kalman Filters could fail here too if linearized dynamics has positive Lyapunov exponents especially for Burgers equations which have only zero Lyapunov exponents [5]. Our design does not rely upon linearization. Instead we make use of the intrinsic symmetries provided by homogeneity of the state equation in combination with rather mild uniform observability assumptions along observer's trajectory to design a new homogeneous observer which together with the proof of existence and uniqueness of solutions for the system of nonlinear differential equations, which describe dynamics of the observer and its gain, represent our key contribution.

The proposed design relies upon an important feature of the matrix differential equation, describing observer's gain, yet another important contribution, namely the practical fixed-time convergence (see e.g. [10]) of the gain, which means that independently of the initial condition the gain converges to a neighbourhood of zero in a fixed time. We demonstrate that the uniform complete observability [11] along the observer trajectory is sufficient for fixed time convergence. We also stress that standard tools of robust design, e.g. various growth estimates for LTV systems, or invariance of uniformly exponentially convergent LTVsystems to Lipschitz perturbations [12] could turn out to be very conservative for the considered systems (see remarks in Section IV-C).

Finally we stress that our design is minimal in that one cannot make use of Lyapunov subspace reduction (e.g. [5], [13]) and restrict the observer's gain corrections just to the stable Lyapunov subspace due to the following fact: all the Lyapunov exponents of the considered dynamical system equal to zero. Nevertheless, unlike [5] we do not require to observe all "unstable directions" instead requiring the uniform complete observability along the observer's trajectory. Design of finite-time homogeneous observers for different classes of homogeneous systems was discussed in [14], [15]. 
The generalized homogeneous dynamical systems where introduced in [16].

\section{MATHEMATICAL PRELIMINARIES}

a) Notation: $\mathbb{R}^{n}$ - Euclidean space of $n$-dimensional column vectors with real-valued entries and canonical basis $\left\{\psi_{1} \ldots \psi_{n}\right\} ; C\left(t_{0}, T, \mathbb{R}^{n}\right)$ - the space of continuous $\mathbb{R}^{n}$ valued functions; $\mathbb{S}^{n}$ - Hilbert space of symmetric nonnegative definite $n \times n$-matrices. $\lambda_{\min }(P)$ and $\lambda_{\max }(P)$ denote minimal and maximal eigen-values of a matrix $P$. $\operatorname{Tr}(P)$ - trace of $P ; I_{n} \in \mathbb{S}^{n}-$ the $n \times n$-identity matrix;

b) Trace Inequalities: If $A$ is symmetric and $B$ is skewsymmetric then $\operatorname{Tr}\left(A B+B^{\top} A\right)=0$.

Lemma 1: If $0<A \in \mathbb{S}^{n}$ then

$$
\|A\|_{\infty}=\max _{i j}\left|a_{i j}\right| \leq \operatorname{Tr} A \leq \sqrt{n \operatorname{Tr} A^{2}}
$$

c) Fixed-time convergence: Recall [10] that $\Omega \subset \mathbb{R}^{n}$ is said to be globally uniformly fixed-time attractive set of the system

$$
\dot{\xi}(t)=f(t, \xi(t)), \quad t>t_{0}
$$

if there exists $T_{\max }>0$ such that

$$
\xi(t) \in \Omega, \quad \forall t \geq t_{0}+T_{\max }
$$

for any initial value $\xi\left(t_{0}\right) \in \mathbb{R}^{n}$ and any $t_{0} \in \mathbb{R}$.

\section{PRoblem Statement}

Let $x(t) \in \mathbb{R}^{n}$ and $y(t) \in \mathbb{R}^{m}$ denote the state vector and output of the following system:

$$
\begin{aligned}
& \dot{x}(t)=B(x(t)) x(t), \quad x\left(t_{0}\right)=x_{0}, \\
& y(t)=C(t) x(t)
\end{aligned}
$$

provided $x \mapsto B(x) \in \mathbb{R}^{n \times n}$ is a linear matrix-valued mapping such that $B(x)=-B^{\top}(x)$ and, $C(t) \in \mathbb{R}^{m \times n}$ is a given measurable matrix-valued function such that

$$
\lambda_{\max }\left(C^{\top}(t) C(t)\right) \leq \bar{c}<+\infty .
$$

Consider the following system of equations:

$$
\begin{array}{ll}
\dot{z}=B(z) z+P C^{\top} R(y-C z), & z\left(t_{0}\right)=0 \\
\dot{P}=B(z) P+P B^{\top}(z)-P C^{\top} R C P+Q(y), & P\left(t_{0}\right)=0
\end{array}
$$

where $R=R^{\top}$ and $Q=Q^{\top}$ are given continuous matrixvalued functions such that:

$$
\begin{aligned}
& 0<\underline{r} \leq \lambda_{\min }(R(t)) \leq \lambda_{\max }(R(t)) \leq \bar{r}<+\infty, \\
& Q(t)=\underline{q}\|y(t)\|^{2} I, \quad \underline{q}>0 .
\end{aligned}
$$

In the forthcoming sections we report the following results:
- existence and uniqueness of the continuous solution for the system of nonlinear differential eqs. (2), (4) and (5) on $\left[t_{0}, T\right)$ for any real $T>t_{0}$,

- sufficient conditions of fixed-time convergence for the gain $P$ solving the non-linear matrix differential eq. (5),

- sufficient conditions of asymptotic convergence of $z(t)$, the solution of eq. (4) to $x(t)$ for any $x\left(t_{0}\right) \in \mathbb{R}^{n}$ verifying natural observability conditions.

\section{A. Motivating examples}

As noted in Section I the class of dynamical systems studied here includes finite dimensional projections of some important PDEs including Burgers(-Hopf) equation (in 1D) and Euler equations (in 2D).

Following [5], [6] we consider the ODE obtained by discretizing the Burgers(-Hopf) equation $u_{t}=-\frac{\partial_{\xi} u^{2}}{2}$ on $(0,1)$ with periodic boundary conditions by using the finite difference scheme:

$$
\dot{u}_{i}=-\frac{n}{6}\left(u_{i}\left(u_{i+1}-u_{i-1}\right)+\left(u_{i+1}^{2}-u_{i-1}^{2}\right)\right)
$$

taken on a periodic lattice $\left(i=1 \ldots n, u_{-1}=u_{n}, u_{n+1}=\right.$ $u_{1}$ ) which has the properties that

- the quadratic energy $\sum_{i} u_{i}^{2}$ is conserved, implying that every sphere in $\mathbb{R}^{n}$ is invariant under the motion of the system and $\|u\|$ is constant,

- the trace of the Jacobian of the r.h.s. of (8) is zero, implying that the flow conserves the volume of the phase element.

Denoting $x=\left(u_{1} \ldots u_{n}\right)^{\top}$ and setting $D=\left\{D_{i j}\right\}_{i, j=1}^{n}$ with $D_{j i}=-D_{i j}$ for $j \leq i$, and $D_{i i+1}=1, D_{i j}=0$ for $j>i+1$ except for $D_{1 n}=-1$, we can rewrite (8) as follows: $\dot{x}=B(x) x$ with

$$
B(x)=-\frac{n}{6}(\operatorname{diag}(x) D+D \operatorname{diag}(x)) .
$$

Clearly,

$$
B^{\top}(x)=-\frac{n}{6}\left(D^{\top} \operatorname{diag}(x)+\operatorname{diag}(x) D^{\top}\right)=-B(x)
$$

since $D^{\top}=-D$.

Euler $^{1}$ equations in $2 \mathrm{D}$

$$
\begin{aligned}
& \partial_{t} \omega+\vec{u} \cdot \nabla \omega=0, \quad-\Delta \psi=\omega, \\
& \vec{u}=\bar{u}+\nabla^{\perp} \psi, \omega(0)=\operatorname{curl}\left(\vec{u}_{0}\right),
\end{aligned}
$$

with periodic boundary conditions at the boundary of a rectangular domain can be approximated by an ODE which has similar properties. Namely, assuming periodic boundary conditions, and applying Fourier-Galerkin (FG) approximation one can project Euler equation onto a $2 N+1$-dimensional subspace generated by $\left\{e^{i k x} e^{i s y}\right\}_{|k|,|s| \leq \frac{N}{2}}$ and obtain an ODE for the projection coefficients in the form $\dot{x}=B(x) x$ with skew-symmetric linear (in $x$ ) matrix $B$. In this case the

\footnotetext{
${ }^{1}$ More specifically, vorticity-streamfunction formulation of Euler equation
} 
components of $x$ will represent projection coefficents of the solution. Analogously, for homogeneous Dirichlet conditions one can take sin-basis. We refer the reader to [3] for further details.

\section{MAIN RESUltS}

\section{A. Existence and uniqueness of continuous solutions}

Theorem 1: For any $x\left(t_{0}\right)=x_{0} \in \mathbb{R}^{n}$ and any $T \in$ $\left(t_{0},+\infty\right)$ the system of eqs. (2), (4) and (5) has the unique solution $(x, z, P)$ such that $x, z \in C\left(t_{0}, T, \mathbb{R}^{n}\right)$ and $P \in$ $C\left(t_{0}, T, \mathbb{S}^{n}\right)$. Moreover, $P(t)>0$ for all $t \in\left(t_{1}, T\right)$, where $t_{1} \geq t_{0}$ is an instant of time such that $y(t)=0$ for $t \leq t_{1}$ and $y\left(t_{1}+\delta\right) \neq 0$ for a small $\delta>0$.

Proof: Since $B$ is skew-symmetric then $\dot{x}^{\top}(t) x(t)=0$, i.e. $\|x(t)\|=\left\|x\left(t_{0}\right)\right\|=\theta<+\infty$ and $\|y(s)\| \leq \bar{c} \theta<+\infty$ for all $t \geq t_{0}$. Taking into account that the right-side of the system (4)-(5) is locally Lipschitz continuous w.r.t. state variables and continuous w. r. t. to time variable, we conclude (e.g. by using standard argument based on Picard theorem) that this system has the unique solution $(z, P)$ defined on a time interval $\left[t_{0}, T\right)$ such that $P(t)$ is a symmetric matrix for all $t \geq t_{0}$.

If $y(t)=0$ for all $t \leq t_{1}$ then $Q(y(t))=0, P(t)=0$ and $z(t)=0$ for all $t \leq t_{1}$. However, $\lim _{\delta \rightarrow 0^{+}} \dot{P}\left(t_{1}+\delta\right)=$ $\lim _{\delta \rightarrow 0^{+}} Q\left(y\left(t_{1}+\delta\right)\right)>0$, i.e. $P(t)>0$ for $t>t_{1}$.

Let us show that $0<P(t)=P^{\top}(t)<a I, a<+\infty$ for all $t \in\left(t_{1}, T\right)$, where $T>t_{1}$ is given by one of the following cases:

1) $T=+\infty$;

2) $T<+\infty, \sup _{t \in\left[t_{1}, T\right)}\|z(t)\|<+\infty$ and $\|P(t)\| \rightarrow$ $+\infty$ as $t \rightarrow T$

3) $T<+\infty,\|z(t)\| \rightarrow+\infty$ as $t \rightarrow T$ and $\sup _{t \in\left[t_{1}, T\right)}\|P(t)\|<+\infty$;

4) $T<+\infty,\|z(t)\| \rightarrow+\infty$ and $\|P(t)\| \rightarrow+\infty$ as $t \rightarrow T$;

5) $T<+\infty, \sup _{t \in\left[t_{1}, T\right)}\|z(t)\|<+\infty, \sup _{t \in\left[t_{1}, T\right)}\|P(t)\|<+\infty$ and $P(T)$ is not positive definite.

Let us now prove that case 1) is the only possible one, i.e. $T=+\infty$ for any $z_{0} \in \mathbb{R}^{n}$ and $P_{0}>0$. Suppose the contrary.

Case 2). In this case, $\|B(z)\| \leq\|B\|\|z\|_{\infty}<+\infty$ and we can write (recall that $\operatorname{Tr}\left(B(z) P+P B^{\top}(z)\right)=0$ ):

$$
\frac{d}{d t} \operatorname{Tr}(P)=-\operatorname{Tr}\left(P C^{\top} R C P\right)+\operatorname{Tr}(Q)
$$

and

$$
\operatorname{Tr} P(t) \leq n \int_{t_{0}}^{T}\|y(s)\|^{2} d s<+\infty, \quad \forall t \in\left[t_{1}, T\right] .
$$

Hence, using Lemma 1 we derive the contradiction.

Case 3). If

$$
V_{0}=z^{\top} P^{-1} z
$$

then for $t>t_{1}$ we have

$$
\begin{aligned}
\dot{V}_{0}= & -z^{\top} C^{\top} R C z+2 z^{\top} C^{\top} R C x-z^{\top} P^{-1} Q P^{-1} z^{\top} \\
= & -z^{\top} C^{\top} R C z+2 z^{\top} C^{\top} R C x-(x)^{\top} C^{\top} R C x+ \\
& x^{\top} C^{\top} R C x-z^{\top} P^{-1} Q P^{-1} z^{\top} \\
= & -(z-x)^{\top} C^{\top} R C(z-x)+x^{\top} C^{\top} R C x-z^{\top} P^{-1} Q P^{-1} z \\
\leq & x^{\top} C^{\top} R C x .
\end{aligned}
$$

Hence $V_{0}(T) \leq V_{0}(T-\varepsilon)+\bar{r} \bar{c} \theta^{2} \varepsilon$, where $0<\varepsilon<T-$ $t_{1}$. By assumption, $\|P(t)\|$ is uniformly bounded on $[T-$ $\varepsilon, T)$. Hence, using $V_{0}(z(T))<+\infty$ we conclude $\|z(T)\|<$ $+\infty$.

Case 4). Let $V$ be defined as follows

$$
V(z, P)=z^{\top} P^{-1} z+\operatorname{Tr}(P) .
$$

We have:

$$
\begin{aligned}
\dot{V}= & -z^{\top} C^{\top} R C z+2 z^{\top} C^{\top} R C x-z^{\top} P^{-1} Q P^{-1} z^{\top}+ \\
& \operatorname{Tr}(Q)-\operatorname{Tr}\left(P C^{\top} R C P\right) \\
= & -(z-x)^{\top} C^{\top} R C(z-x)+x^{\top} C^{\top} R C x+ \\
& \operatorname{Tr}(Q)-z^{\top} P^{-1} Q P^{-1} z^{\top}-\operatorname{Tr}\left(P C^{\top} R C P\right) \\
\leq & x^{\top} C^{\top} R C x+\operatorname{Tr}(Q)
\end{aligned}
$$

and

$V(t) \leq V(T-\varepsilon)+\underline{q} \int_{T-\varepsilon}^{T}\|y\|^{2} d s+\int_{T-\varepsilon}^{T} x^{\top} C^{\top} R C x d s<+\infty$

for all $t \in[T-\varepsilon, T]$, where $0<\varepsilon<T-t_{1}$. Using Lemma 1 we derive the contradiction.

Case 5). By assumption, $P(t)>0$ on $\left(t_{1}, T\right)$ and $P(T) x=$ 0 for some $x \neq 0$. Hence, $W=P^{-1}(t)$ is defined for every $t \in\left(t_{1}, T\right)$, and $\lim _{t \uparrow T}\|W(t)\|=+\infty$. In this case, $\operatorname{Tr} W(t) \rightarrow \infty$ as $t \rightarrow T$ due to Lemma 1 . For any $t \in\left[t_{0}, T\right)$ we have:

$\dot{W}=-W \dot{P} W=-B^{\top}(z) W-W B(z)-W Q W+C^{\top} R C$, $W\left(t_{0}\right)=P^{-1}\left(t_{0}\right)$. Since $\operatorname{Tr}\left(W B(x)+B^{\top}(x) W\right)=0$ then

$$
\frac{d}{d t} \operatorname{Tr} W=\operatorname{Tr}\left(C^{\top} R C\right)-\operatorname{Tr}(W Q W) \leq \bar{r} \bar{c} n .
$$

Hence, $\operatorname{Tr}(W(t))$ cannot tend to $\infty$ as $t \rightarrow T$. We derive the contradiction.

The following trivial corollary is derived from Theorem (1).

Corollary 1: Theorem remains true for $P\left(t_{0}\right)>0$ and $t_{1}=$ $t_{0}$ in this case.

\section{B. Fixed-time convergence for the gain $P$}

Now, let us demonstrate practical fixed-time convergence of the gain $P$. To this end we introduce

Definition 1: Let $z$ solve (4), and let $\dot{X}=B(z) X, X(0)=$ $X_{0}$, and set $\Phi(t, s)=X(t) X^{-1}(s) . \mathcal{N}(t, t-\sigma ; R, z)$ is said 
to be a gramian along a trajectory $z$ of (4) if $\mathcal{N}(t, t-$ $\sigma ; R, z)=\int_{t-\sigma}^{t} \Phi^{\top}(s, t) C^{\top} R C \Phi(s, t) d s$.

Corollary 2: Let $z$ and $P$ solve (4)-(5) on $\left[t_{0},+\infty\right)$, and let $\mathcal{N}(t, t-\sigma ; R, z)$ denote the gramian along the trajectory $z$. If there exist $\alpha>0$ and $\sigma>0$ such that for any $t>t_{0}+\sigma$ we have that $\alpha I<\mathcal{N}(t, t-\sigma ; I, z)$ then

$$
\lambda_{\max }(P(t)) \leq \frac{1}{\underline{r} \alpha}+\underline{q} \int_{t-\sigma}^{t}\|y(s)\|^{2} d s, \quad t \geq t_{0}+\sigma .
$$

Proof: Assume that $t<+\infty$ and consider the standard LQR design problem for the system $\dot{q}=-B^{\top}(z) q+C^{\top} u$, $q(t)=h$ with the cost $J(u)=q^{\top}\left(t_{0}\right) P\left(t_{0}\right) q\left(t_{0}\right)+$ $\int_{t_{0}}^{t} u^{\top} R^{-1} u+q^{\top} Q q d s$. It is known that the feed-back $\hat{u}=$ $R C P q$ minimizes $J$ and $J(\hat{u})=h^{\top} P(t) h$. Hence for any other control $u$ we have that $J(\hat{u})=h^{\top} P(t) h \leq J(u)$. Let us select $u$ as follows: set $u(s)=R C(s) \Phi(s, t) \mathcal{N}^{-1}(t, t-$ $\sigma ; R, z) h$ for $s \in[t-\sigma, t]$ and set $u(s)=0$ for $s \in\left[t_{0}, t-\sigma\right)$. Let us show that for this $u$ one gets: $q(s)=0$ for all $s \in\left[t_{0}, t-\sigma\right]$. To this end recall that

$$
q(s)=\Phi^{\top}(t, s) h-\int_{s}^{t} \Phi^{\top}(\tau, s) C^{\top}(\tau) u(\tau) d \tau
$$

and so $q(t-\sigma)=0$ if $^{2}$

$$
\Phi^{\top}(t, t-\sigma) h=\Phi^{\top}(t, t-\sigma) \int_{t-\sigma}^{t} \Phi^{\top}(\tau, t) C^{\top}(\tau) u(\tau) d \tau
$$

Clearly, for the above choice of $u$ we get that $q(s)=0$ for all $s \in\left[t_{0}, t-\sigma\right]$. Hence, for this $u$ we get that

$$
\begin{aligned}
J(\hat{u}) & =h^{\top} P(t) h \leq \int_{t-\sigma}^{t} u^{\top} R^{-1} u+q^{\top} Q q d s \\
& =h^{\top} \mathcal{N}^{-1}(t, t-\sigma ; R, z) h+\int_{t-\sigma}^{t} q^{\top} Q q d s \\
& \leq \frac{\|h\|^{2}}{\underline{r} \alpha}+\int_{t-\sigma}^{t} q^{\top} Q q d s
\end{aligned}
$$

since $\mathcal{N}(t, t-\sigma ; R, z) \geq \underline{r} \mathcal{N}(t, t-\sigma ; I, z) \geq \underline{r} \alpha$. To compute $\int_{t-\sigma}^{t} q^{\top} Q q d s$ we first note that

$$
\begin{aligned}
q(s)= & \Phi^{\top}(t, s)\left(I-\mathcal{N}(t, s ; R, z) \mathcal{N}^{-1}(t, t-\sigma ; R, z)\right) h= \\
& \Phi^{\top}(t, s) \mathcal{N}(s, t-\sigma ; R, z) \mathcal{N}^{-1}(t, t-\sigma ; R, z) h
\end{aligned}
$$

for $s \geq t-\sigma$ and so

$$
\begin{gathered}
\int_{t-\sigma}^{t} q^{\top} Q q d s= \\
h^{\top} \mathcal{N}^{-1}(t, t-\sigma ; R, z) W(t, t-\sigma) \mathcal{N}^{-1}(t, t-\sigma ; R, z) h
\end{gathered}
$$

with

$$
\begin{gathered}
W(t, t-\sigma)= \\
\int_{t-\sigma}^{t} \mathcal{N}(s, t-\sigma ; R, z) \Phi(t, s) Q \Phi^{\top}(t, s) \mathcal{N}(s, t-\sigma ; R, z) d s
\end{gathered}
$$

${ }^{2}$ We used the obvious equality $\Phi(\tau, t-\sigma)=\Phi(\tau, t) \Phi(t, t-\sigma)$
Recalling that $\Phi(t, s) \Phi^{\top}(t, s)=I$ for skew-symmetric systems we get that:

$$
W(t, t-\sigma) \leq \int_{t-\sigma}^{t} \lambda_{\max }(Q) \mathcal{N}^{2}(s, t-\sigma ; R, z) d s .
$$

Finally, we note that $\mathcal{N}^{2}(s, t-\sigma ; R, z) \leq \mathcal{N}^{2}(t, t-\sigma ; R, z)$ hence $W(t, t-\sigma) \leq \mathcal{N}^{2}(t, t-\sigma ; R, z) \int_{t-\sigma}^{t} \lambda_{\max }(Q) d s$ and so $\int_{t-\sigma}^{t} z^{\top} Q z d s \leq\|h\|^{2} \int_{t-\sigma}^{t} \lambda_{\max }(Q) d s$. This and (12) completes the proof.

The straightforward consequence of Corollary 2 is practical fixed-time convergence of the Riccati equation.

Corollary 3: Let the conditions of Corollary 2 hold true. Then

$$
\Omega=\left\{P \in \mathbb{S}^{n}: 0<P \leq\left(\underline{q} \bar{c} \sigma\left\|x_{0}\right\|^{2}+(\underline{r} \alpha)^{-1}\right) I\right\}
$$

is globally uniformly fixed-time attractive set of the system (5) with $T_{\max }=\sigma$.

By using the same LQR-based argument as in the proof of Corollary 2 one can demonstrate the practical fixed-time convergence for $P^{-1}$ :

Corollary 4: Let $z$ and $P$ solve (4)-(5) on $\left[t_{0},+\infty\right)$. If there exists $\tilde{\sigma}>0$ such that

$$
\int_{t-\tilde{\sigma}}^{t}\|y(s)\|^{2} d s \geq \alpha_{y}>0
$$

then

$$
\lambda_{\max }\left(P^{-1}(t)\right) \leq \frac{1}{\alpha_{y} \underline{q}}+\tilde{\sigma} \bar{c} \bar{r}, \quad \forall t \geq t_{0}+\tilde{\sigma} .
$$

Proof: Given $z$ let us denote

$$
\mathcal{N}(t, s, Q)=\int_{s}^{t} \Phi(t, \tau) Q(y(\tau)) \Phi^{\top}(t, \tau) d \tau .
$$

Since $\Phi$ is orthogonal matrix $(B(z)$ is skew-symmetric!) it follows that $\mathcal{N}(t, s, Q)=\int_{s}^{t}\|y(\tau)\|^{2} d \tau$. By theorem 1 there is $t^{*} \in\left(t_{0}, T\right)$ such that $P\left(t^{*}\right)>0$. Consider the following standard LQR control problem

$$
\begin{gathered}
\dot{q}=B(z) q+Q^{\frac{1}{2}}(y) u, \quad q(t)=h \in \mathbb{R}^{n}, \\
J(u)=q\left(t^{*}\right)^{\top} P^{-1}\left(t^{*}\right) q\left(t^{*}\right)+\int_{t^{*}}^{t} u^{\top} u+q^{\top} C^{\top} R C q d \tau .
\end{gathered}
$$

Since $W(t)=P^{-1}(t)$ satisfies

$\dot{W}=-B^{\top}(z) W-W B(z)-W Q W+C^{\top} R C, W\left(t^{*}\right)=P^{-1}\left(t^{*}\right)$

then $\tilde{u}=Q^{\frac{1}{2}} W q$ minimizes the functional $J$ and $J(\hat{u})=h^{\top} W(t) h$. For any other control $u$ we have $J(\hat{u}) \leq J(u)$. Let us select $u$ as follows: set $u(s)=$ $Q^{1 / 2}(s) \Phi^{\top}(s, t) \mathcal{N}^{-1}(t, t-\tilde{\sigma}, Q) h$ for $s \in[t-\tilde{\sigma}, t]$ and $u(s)=0$ for $s \in\left[t^{*}, t-\tilde{\sigma}\right]$. Let us show that for this $u$ we have $q(s)=0$ for all $s \in\left[t^{*}, t-\tilde{\sigma}\right]$. Since for $s \in[t-\tilde{\sigma}, t]$ we have

$$
q(s)=\Phi(t, s) h-\int_{s}^{t} \Phi(s, \tau) Q^{1 / 2}(\tau) u(\tau) d \tau=
$$




$$
\begin{gathered}
\Phi(t, s)\left(I_{n}-\int_{s}^{t} \Phi(t, \tau) Q(\tau) \Phi^{\top}(\tau, t) d \tau \mathcal{N}^{-1}(t, t-\tilde{\sigma}, Q)\right) h= \\
\Phi(t, s)\left(I_{n}-\mathcal{N}(t, s, Q) \mathcal{N}^{-1}(t, t-\tilde{\sigma}, Q)\right) h= \\
\Phi(t, s)(\mathcal{N}(t, t-\tilde{\sigma}, Q)-\mathcal{N}(t, s, Q)) \mathcal{N}^{-1}(t, t-\tilde{\sigma}, Q) h= \\
\Phi(t, s) \mathcal{N}(s, t-\tilde{\sigma}, Q) \mathcal{N}^{-1}(t, t-\tilde{\sigma}, Q) h
\end{gathered}
$$

then $q(s)=0$ for $s \in\left[t^{*}, t-\tilde{\sigma}\right]$. Hence,

$$
\begin{gathered}
\int_{t^{*}}^{t} q^{\top} C^{\top} R C q d s= \\
h^{\top} \mathcal{N}^{-\top}(t, t-\tilde{\sigma}, Q) V(t, t-\tilde{\sigma}) \mathcal{N}^{-1}(t, t-\tilde{\sigma}, Q) h
\end{gathered}
$$

where

$$
\begin{gathered}
V(t, t-\tilde{\sigma})= \\
\int_{t-\tilde{\sigma}}^{t} \mathcal{N}(s, t-\tilde{\sigma}, Q) \Phi^{\top}(t, s) C^{\top} R C \Phi(t, s) \mathcal{N}(s, t-\tilde{\sigma}, Q) d s .
\end{gathered}
$$

Since $\Phi^{\top}(t, s) \Phi(t, s)=I$ for skew-symmetric matrices then

$$
V(t, t-\tilde{\sigma}) \leq \bar{r} \bar{c} \int_{t-\tilde{\sigma}}^{t} \mathcal{N}^{2}(s, t-\tilde{\sigma}, Q) d s
$$

Taking into account $\mathcal{N}^{2}(s, t-\tilde{\sigma}, Q) \leq \mathcal{N}^{2}(t, t-\tilde{\sigma}, Q)$ we derive

$$
\int_{t^{*}}^{t} q^{\top} C^{\top} R C q d s \leq \tilde{\sigma} \bar{c} \bar{r}\|h\|^{2} .
$$

On the other hand, we have

$$
\begin{gathered}
\int_{t^{*}}^{t} u^{\top} u d s= \\
h^{\top} \mathcal{N}^{-1}(t, t-\tilde{\sigma}, Q) \int_{t-\tilde{\sigma}}^{t} \Phi(s, t) Q(s) \Phi^{\top}(s, t) d s \mathcal{N}^{-1}(t, t-\tilde{\sigma}, Q) h
\end{gathered}
$$

and

$$
\int_{t^{*}}^{t} u^{\top} u d s=h^{\top} \mathcal{N}^{-1}(t, t-\tilde{\sigma}, Q) h \leq \frac{\|h\|^{2}}{\alpha_{y} \underline{q}} .
$$

Hence, we derive

$$
J(\hat{u})=h^{\top} W(t) h \leq J(u) \leq \frac{\|h\|^{2}}{\alpha_{y} \underline{q}}+\tilde{\sigma} \bar{c} \bar{r}\|h\|^{2}, \quad \forall h \in \mathbb{R}^{n} .
$$

Remark 1: Note that for the case of observable LTI systems the corresponding Riccati equation (5) has the property of the practical fixed-time convergence as defined above in 3 .
C. Asymptotic convergence of the observer $z$

Let us define $B_{1}(x)=\left[\begin{array}{lll}B\left(\psi_{1}\right) x & \ldots & B\left(\psi_{n}\right) x\end{array}\right]$ and

$$
W(x, t)=P^{\frac{1}{2}}(t) B_{1}^{\top}(x) P^{-\frac{1}{2}}(t)+P^{-\frac{1}{2}}(t) B_{1}(x) P^{\frac{1}{2}}(t),
$$

and $Z=P^{\frac{1}{2}} C^{\top} R C P^{\frac{1}{2}}-\underline{q}\|y\|^{2} P^{-1}$.

Theorem 2: Let $C$ and $R$ be constant matrices. Let $x$ solve (2), and $z, P$ solve (4)-(5). Define $e=x-z$ and $V(t)=\left\|P^{-\frac{1}{2}}(t) e(t)\right\|^{2}$. If $x\left(t_{0}\right)=x_{0}$ is so that there exists $\sigma_{\left\|x_{0}\right\|}>0$ such that $\forall t>t_{0}+\sigma_{\left\|x_{0}\right\|}$ :

$$
\int_{t-\sigma_{\left\|x_{0}\right\|}}^{t} \lambda_{\max }(W(x(s), s)-Z(s)) d s<0,
$$

then $V(t) \rightarrow 0$ for every $x\left(t_{0}\right)=\lambda x_{0}, \lambda>0$. If in addition $\lambda_{\max }(P(t))<\bar{p}<+\infty$, e.g. if conditions of corollary 2 hold, then $\|x(t)-z(t)\| \rightarrow 0$ for every $\lambda x_{0}, \lambda>0$.

Proof: Upon differentiating $e$ we find: $\dot{e}=B(x) x-$ $B(z) z-P C^{\top} R(y-C z)=\left(B(z)-P C^{\top} R C\right) e+B(e) x$. Recalling that $\dot{P}^{-1}=-P^{-1} \dot{P} P^{-1}$ and using (5) we find that

$\dot{V}(e, t)=2\left(P^{-1} e, B(e) x\right)-(R C e, C e)-q_{0}\|y(t)\|^{2}\left\|P^{-1} e\right\|^{2}$

Using the definitions of $W$ and $Z$ we get:

$$
2\left(P^{-1} e, B(e) x\right)=\left(\left(B_{1}^{\top}(x) P^{-1}+P^{-1} B_{1}(x)\right) e, e\right)
$$

so that $2\left(P^{-1} e, B(e) x\right)=\left(W P^{-\frac{1}{2}} e, P^{-\frac{1}{2}} e\right)$ and

$$
\dot{V}(e, t)=\left((W-Z) \frac{P^{-\frac{1}{2}} e}{V^{\frac{1}{2}}}, \frac{P^{-\frac{1}{2}} e}{V^{\frac{1}{2}}}\right) V
$$

Since $\left\|\frac{P^{-\frac{1}{2}} e}{V^{\frac{1}{2}}}\right\|=1$ for any $e(t)$ and $P(t)$ we get that

$$
\left((W-Z) \frac{P^{-\frac{1}{2}} e}{V^{\frac{1}{2}}}, \frac{P^{-\frac{1}{2}} e}{V^{\frac{1}{2}}}\right) \leq \lambda_{\max }(W-Z)
$$

This demonstrates that $\dot{V} \leq \lambda_{\max }(W-Z) V$. By Gronwall lemma we get:

$$
V(t) \leq e^{\int_{t-\sigma_{\left\|x_{0}\right\|}}^{t} \lambda_{\max }(W(x(s), s)-Z(s)) d s} V\left(t-\sigma_{\left\|x_{0}\right\|}\right)
$$

Thus eq. (14) implies $V(t) \rightarrow 0$ but this convergence is not necessarily monotone. To demonstrate that $V(t) \rightarrow 0$ for every $x\left(t_{0}\right)=\lambda x_{0}, \lambda>0$ we note that eqs. (2), (4) and (5) taken together form a homogeneous system: $\dot{\xi}=F(\xi)$, $F(\lambda \xi)=\lambda^{2} F(\xi)$ where $\xi=(x, z, P)$. Hence $\xi\left(t, \lambda \xi_{0}\right)=$ $\lambda \xi\left(\lambda t, \xi_{0}\right)$, where $\xi(\cdot, \bar{\xi})$ is a solution of $\dot{\xi}=F(\xi)$ with the initial condition $\xi(0)=\bar{\xi}$. Since $\xi_{0}=\left(x_{0}, 0,0\right)$ (recall eqs. (2), (4) and (5)) it follows that $\lambda \xi_{0}=\left(\lambda x_{0}, 0,0\right)$ and so $V(t)=\left\|P^{-\frac{1}{2}}(\lambda t, 0)\left(x\left(\lambda t, x_{0}\right)-z(\lambda t, 0)\right)\right\|^{2} \rightarrow 0$ for any $\lambda>0$ since, as we saw above, it holds for $x_{0}$.

If $\lambda_{\max }(P(t))<\bar{p}<+\infty$ then $P^{-1}(t)>p_{1}>0$ for all $t>$ $t_{0}$ and hence $V(t) \rightarrow 0$ implies $\|e(t)\| \rightarrow 0$. In particular, conditions of corollary 2 imply that $P^{-1}(t)>p_{1}>0$ for all $t>t_{0}$. 
The above result do not provide means to check the convergence of the observer numerically. In what follows we provide a sufficient condition which relies upon splitting the state vector $x$ into observable and unobservable parts, i.e. $x=x^{u} \oplus x^{o}, x^{o}=C^{+} y, x^{u}=x-x^{o}$, and noting that $\left\|x^{u}(t)\right\|^{2}=\theta^{u}(t)$ provided $\theta^{u}(t)=\|x(t)\|^{2}-\left\|x^{o}(t)\right\|^{2}=$ $\left\|x_{0}\right\|^{2}-\left\|C^{+} y(t)\right\|^{2}$, where $C^{+}$denotes the pseudo-inverse matrix to $C$. It then follows that $x^{u}(t)=\sum_{j=1}^{N^{u}} \alpha_{j} \theta^{u}(t) v_{j}$ where $\alpha_{j} \geq 0, \sum_{j} \alpha_{j}=1$, and the vectors $v_{j}$ are taken as vertices of a polytope $\mathcal{P}$ containing $\{x:\|x\|=1, C x=0\}$, the projection of the unit ball of $\mathbb{R}^{n}$ onto the null-space of $C$. If $C=\left[I_{m}, 0_{m, n-m}\right]$ then the most trivial example of such polytop $\mathcal{P}$ is given by convex combinations of vectors $v_{j}=\left(0 \ldots 0, \tilde{v}_{j}\right)^{\top}$ with $\tilde{v}_{j}$ being $j$ th vertex of the $n-m$ hypercube $[-1,1]^{n-m}$ so that $x_{j}^{u}(t) \in \theta^{u}(t)[-1,1]$. A nontrivial example of $\mathcal{P}$ is given in Section V. Clearly, the tighter $\mathcal{P}$ approximates the projected unit ball of $\mathbb{R}^{n}$ onto the unobservable subspace given by the null-space of $C$ the more accurate representation for $x^{u}$ one gets. In the following corollary we suggest to evaluate an upper bound of $\lambda_{\max }(\cdot)$ from eq. (14) over a polytope $\mathcal{P}$ to derive a practical way of checking eq. (14).

Corollary 5: Let $C(t) \equiv C, R(t) \equiv R$ and $\left\|x_{0}\right\|=\theta$, and set $\theta^{u}(t)=\theta-\left\|C^{+} y(t)\right\|$. Let $\mathcal{P}$ be a polytope containing the projected ball $\{x:\|x\|=1, C x=0\}$ with vertices $v_{j}$, $j=1, \ldots, N_{u}$. Define

$\bar{g}(t)=\max _{j \in\left\{1 \ldots N_{u}\right\}}\left\{\lambda_{\max }\left(W\left(\theta^{u}(t) v_{j}\right)+W\left(C^{+} y(t)\right)-Z(t)\right)\right\}$ If $\int_{t-\sigma_{\left\|x_{0}\right\|}}^{t} \bar{g}(s) d s<0$ for some $\sigma_{\left\|x_{0}\right\|}>0$ and all $t>$ $t_{0}+\sigma_{\left\|x_{0}\right\|}$ then $V(t) \rightarrow 0$ (non-monotonically!) for every $x\left(t_{0}\right)=\lambda x_{0}, \lambda>0$.

Proof: We first note that, by definition of $\bar{g}(t)$,

$$
\alpha_{i}\left(W\left(\theta^{u}(t) v_{i}, t\right)+W\left(x^{o}(t), t\right)-Z(t)\right) \leq \bar{g}(t) I_{n} .
$$

and thus

$$
\sum_{i=1}^{n} \alpha_{i}\left(W\left(\theta^{u}(t) v_{i}, t\right)+W\left(x^{o}(t), t\right)-Z(t)\right) \leq \bar{g}(t) I_{n} .
$$

On the other hand, recalling that $x=x^{u} \oplus x^{o}$, and that $x^{u}(t)=\sum_{j=1}^{N^{u}} \alpha_{j} \theta^{u}(t) v_{j}$ where $\alpha_{j} \geq 0, \sum_{j} \alpha_{j}=1$, and the vectors $v_{j}$ are the vertices of $\mathcal{P}$, we get:

$W(x, t)-Z(s)=\sum_{i=1}^{n} \alpha_{i}\left(W\left(\theta^{u}(t) v_{i}, t\right)+W\left(x^{o}(t), t\right)-Z(t)\right)$

Hence $\lambda_{\max }(W(x(s), s)-Z(s)) \leq \bar{g}(s)$. Now, the statement follows from theorem 2 .

Remark 2: Note that the estimate of Corollary 5 could turn out to be quite conservative but in many cases including the one considered in the following section it closely follows the $\lambda_{\max }(W-Z)$ computed for the exact $x$.

Remark 3: We note that a more restrictive and thus conservative assumption can be imposed to guarantee convergence of $e$ to 0 : indeed, note that the exact equation for $e, \dot{e}=$
$\left(B(z)-P C^{\top} R C\right) e+B(e) x, e(0)=x_{0}$ can be considered as a perturbed version of $\dot{e}_{1}=\left(B(z)-P C^{\top} R C\right) e_{1}$, which is uniformly exponentially stable if corollary 2 holds, i.e. its fundamental solution $\Phi(t, s)$ verifies $\|\Phi(t, s)\| \leq$ $K e^{-\gamma(t-s)}$. Note that the perturbation, $B(e) x$ is Lipschitz as $B(e) x=B_{1}(x) e$, and $\|x(t)\|=\left\|x_{0}\right\|=\theta$ so that $\left\|B_{1}(x) e\right\| \leq\left\|B_{1}\right\| \theta\|e\|$. By noting that $e(t)=\Phi(t, s) e(s)+$ $\int_{s}^{t} \Phi(t, \tau) B_{1}(x) e(\tau) d \tau$, and applying the integral form of Grownwall lemma one can easily derive that the Lyapunov spectrum of the exact error dynamics is bounded from above by $\gamma-K \theta\left\|B_{1}\right\|$. By using estimates of $\lambda_{\max }(P)$ and $\lambda_{\min }(P)$ obtained in eqs. (11) and (13) one could express $K$ and $\gamma$ in terms of $\underline{q}, \bar{r}$ and some unknown values $\sigma, \alpha$ and then require that $\gamma-K \theta\left\|B_{1}\right\|<0$ to guarantee uniform exponential stability of the error dynamics but it is not clear how to verify this condition numerically without a priori knowledge of $\sigma$ and $\alpha$.

Remark 4: Yet another way to establish stability of the error dynamics is by using Vazhevskii estimates (see [12, p.110]) of the growth of solutions of perturbed LTV systems. Indeed, since $B(z) z=B_{1}(z) z$ one can use $B_{1}(z)$ instead of $B(z)$ in eq. (5). In this case, one would need to change the proofs of the existence and uniqueness theorems given in Section IV-A, but it would still be possible to prove the existence results under some extra assumptions. In the latter case, the error dynamics will change to $\dot{e}=\left(B_{1}(z)-\right.$ $\left.P C^{\top} R C\right) e+B(x) e, e(0)=x_{0}$. Now, Vazhevskii estimate provides

$$
\|e(t)\| \leq\left\|e\left(t_{0}\right)\right\| \exp \left\{\int_{t_{0}}^{t} q(s) d s\right\}
$$

with $q(s)=\lambda_{\max }\left(\left(B_{1}(z)+B(x)-P C^{\top} R C\right)+\left(B_{1}(z)+\right.\right.$ $\left.\left.B(x)-P C^{\top} R C\right)^{\top}\right)$. Since $B(x)=-B^{\top}(x)$ it follows that $q(s)$ is independent of $x$ and $e$ decays to 0 exponentially (but not uniformly!) provided $\int_{t_{0}}^{t} q(s) d s<0$. We stress that this estimate is easy to check numerically since we have all the parameters. However, at least for the system we consider in Section $\mathrm{V}$, we observed that numerically $q(s)$ was always non-negative even though the error $e$ was converging to 0 .

\section{EXPERIMENTAL VALIDATION}

We perform a number of numerical experiments for the ODE obtained by discretizing the Burgers(-Hopf) equation $u_{t}=-\frac{\partial_{\xi} u^{2}}{2}$ on $(0,1)$ with periodic boundary conditions by using the finite difference scheme eq. (8). Recall that this ODE conserves energy and multidimensional volume: $\|x(t)\|=\left\|x_{0}\right\|$ and the Lebesgue measure of the set of initial conditions is preserved by ODE's semigroup.

We set $n=8$ and select $C$ so that $C x=$ $\left(x_{1}, x_{2}, x_{3}, x_{4}, x_{5}, x_{6}\right)^{\top}$, i.e. 3 out of 8 components of $x$ were measured. To perform the simulations, we first generate 10 different "true" initial conditions by drawing 10 vectors $x_{0}$ from a normal distribution, normalizing each so that $\left\|x_{0}\right\|=1$ and removing the mean (to account for the periodicity). Then we set $z(0)=0, P(0)=0, \underline{q}=\bar{r}=$ 


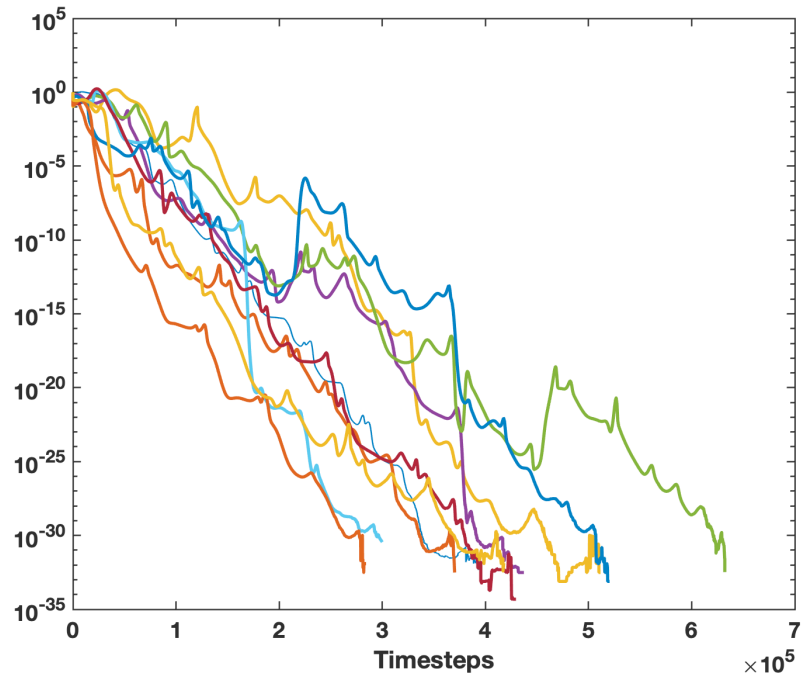

Fig. 1: Convergence of the estimation error $\|x(t)-z(t)\|$ (log-scale) for 10 simulations: for each simulation the intial condition $x_{0} \in \mathbb{R}^{8}$ for the "truth" trajectory was picked randomly and normalized so that $\left\|x_{0}\right\|=1$; the same $z(0)=0, P(0)=0, \underline{q}=\bar{r}=10^{4}$ were used across all simulations.

$10^{4}$, and solve the state equation (2), the filter (4), and Ricatti equation (5) simultaneously by RK4 method with $\Delta t=1.333 \times 10^{-4}$ on a time interval $[0,80]$. Fig. 1 demonstrates the non-monotone character of the convergence of estimation error, and Fig. 2 compares convergence of the estimation error with dynamics of $V$. Both figures justify our choice of sufficient conditions of convergense of integral type: each figure suggests that $V(t)-V(t-\sigma)<0$ and $\|e(t)\|^{2}-\|e(t-\sigma)\|^{2}<0$ for some $\sigma>0$. This intuition is further confirmed in Fig. 3 where we plot $\lambda_{\max }(W-Z)$ : its dynamics suggets that $\int_{t-\sigma}^{t} \lambda_{\max }(W-Z) d s<0$, and indeed the latter holds even for the integral of the worstcase approximation of $\lambda_{\max }(W-Z)$ given by $\bar{g}$ as it is evident from Fig. 3.

To compute $\bar{g}$ we approximated the circle containing the two unobserved components of $x$ by an outer polytope with 40 vertices: we took $N_{u}=40$ points of the form $\tilde{v}_{k}=\left[\cos \left(\frac{2 \pi}{k}\right), \sin \left(\frac{2 \pi}{k}\right)\right]$ on the unit circle and multiplied each by 1.02 to get an outer politope. Then we set $v_{k}=$ $\left(0, \ldots, 0, \tilde{v}_{k}\right)^{\top}, k=1 \ldots 40$ and compute

$\bar{g}(t)=\max _{j \in\left\{1 \ldots N_{u}\right\}}\left\{\lambda_{\max }\left(W\left(\theta^{u}(t) v_{j}\right)+W\left(C^{+} y(t)\right)-Z(t)\right)\right\}$ with $\theta^{u}(t)=\left(1-\left\|C^{\top} C x(t)\right\|^{2}\right)^{\frac{1}{2}}$. Dynamics of $\bar{g}$ closely follows that of $\lambda_{\max }(W-Z)$, and $\int_{t-\sigma}^{t} \bar{g}(s) d s<0$ for a quite large $\sigma=40$, see Fig. 3 .

\section{CONCLUSIONS}

The paper reveals an important relation between uniform complete observability, fixed-time convergence for non-linear

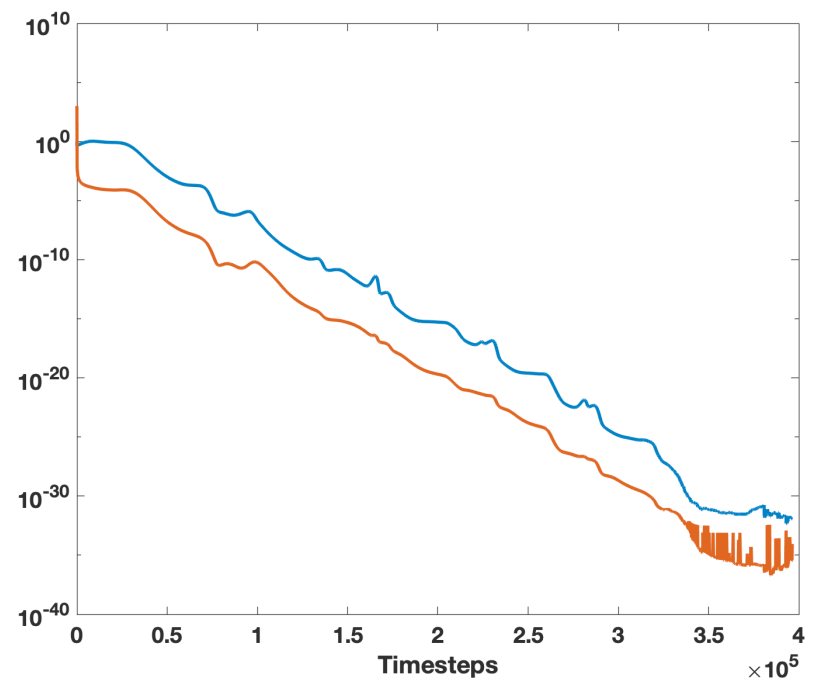

Fig. 2: Convergence of the estimation error (blue) and function $V$ (red).

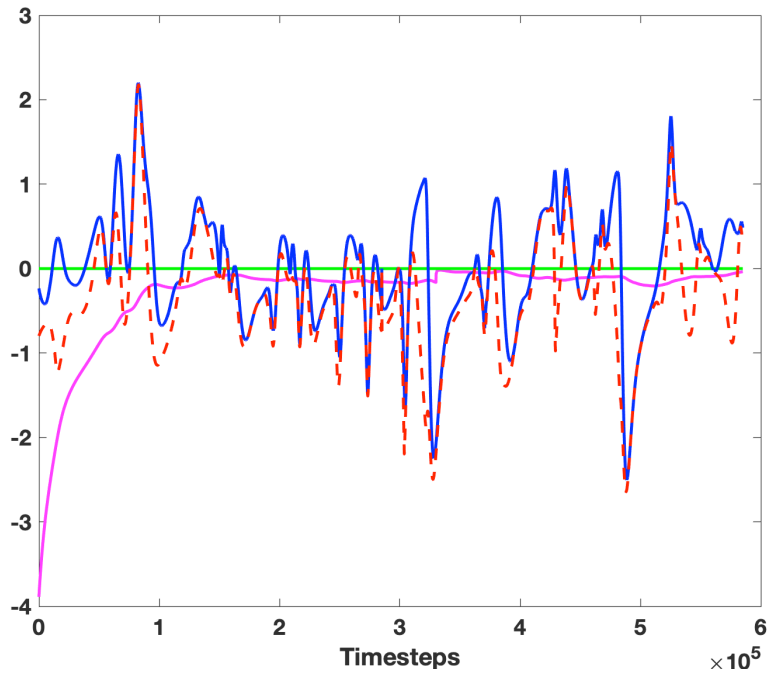

Fig. 3: Comparison of $\lambda_{\max }(W-Z)$ (dashed) with $\bar{g}$ (blue) and the corresponding $\int_{t-\sigma_{\left\|x_{0}\right\|}}^{t} \bar{g}(s) d s$ (magenta) for $\sigma_{\left\|x_{0}\right\|}=40$ on the interval $[0,80]$.

homogeneous matrix differential equations and homogeneous observer design. This relation, on the other hand, requires further exploration of numerically efficient sufficient conditions for convergence, and how to deal with the case of non-trivial model disturbance.

\section{REFERENCES}

[1] R. Stratonovich, "On the theory of optimal non-linear filtering of random functions," Theory of Probability and Its Applications, no. 4, p. 223225 . 
[2] J. Baras, A. Bensoussan, and M. James, "Dynamic observers as asymptotic limits of recursive filters: Special cases," SIAM Journal on Applied Mathematics, vol. 48, no. 5, pp. 1147-1158, 1988.

[3] S. Zhuk, T. T. Tchrakian, and J. Frank, "Exponentially convergent data assimilation algorithm for navier-stokes equations," in 2017 American Control Conference (ACC), May 2017, pp. 3249-3256.

[4] S. Zhuk and T. Tchrakian, "Parameter estimation for euler equations with uncertain inputs," in 2015 54th IEEE Conference on Decision and Control (CDC), Dec 2015, pp. 572-577.

[5] J. Frank and S. Zhuk, "A detectability criterion and data assimilation for nonlinear differential equations," Nonlinearity, vol. 31, no. 11, p. $5235,2018$.

[6] A. Jameson, "Energy estimates for nonlinear conservation laws with applications to solutions of the burgers equation and one-dimensional vicous flow in a shock tube by central difference schemes," in 18th AIAA Computational Fluid Dynamics Conference, 2007, p. 4620.

[7] M. Fliess, J. Lévine, P. Martin, and P. Rouchon, "Flatness and defect of non-linear systems: introductory theory and examples," International journal of control, vol. 61, no. 6, pp. 1327-1361, 1995.

[8] A. Isidori, Nonlinear Control Systems. Springer-Verlag, N. Y. Inc., 1995.

[9] R. Hermann and A. Krener, "Nonlinear controllability and observability," IEEE Transactions on Automatic Control, vol. 22, no. 5, pp. 728-740, October 1977.

[10] A. Polyakov, "Nonlinear feedback design for fixed-time stabilization of linear control systems," IEEE Trans. Autom. Cont., vol. 57(8), pp. 2106-2110, 2012

[11] R. S. Bucy, "The riccati equation and its bounds," Journal of computer and system sciences, vol. 6, no. 4, pp. 343-353, 1972.

[12] L. Y. Adrianova, Introduction to linear systems of differential equations. American Mathematical Soc., 1995.

[13] M. Tranninger, R. Seeber, S. Zhuk, M. Steinberger, and M. Horn, "Detectability analysis and observer design for linear time varying systems," IEEE Control Systems Letters, vol. 4, no. 2, pp. 331-336, 2019 .

[14] V. Andrieu, L. Praly, and A. Astolfi, "Homogeneous Approximation, Recursive Observer Design, and Output Feedback," SIAM Journal of Control and Optimization, vol. 47, no. 4, pp. 1814-1850, 2008.

[15] F. Lopez-Ramirez, A. Polyakov, D. Efimov, and W. Perruquetti, "Finite-time and fixed-time observer design: Implicit Lyapunov function approach," Automatica, vol. 87, no. 1, pp. 52-60, 2018.

[16] A. Polyakov, Generalized Homogeneity in Systems and Control. Springer, 2020. 